\title{
VARIAÇÕES DAS FÁCIES GRANULOMÉTRICAS DOS SEDIMENTOS DE SUPERFÍCIE DE FUNDO DA BAIA DE SANTOS
}

\author{
WALDIR LOPES PONÇANO* e ANTONIO FERNANDO GIMENEZ**
}

\begin{abstract}
Grain-size variations of bottom sediments in Santos Bay were determined for a seven years span of time, through samples collected in 1973 and 1980. The same was carried out on the neighbourhood of Ponta de Itaipu, in this case throughout five periods of sampling along 1974-1975. Results showed no important variations in the grain-size pattern for both cases.
\end{abstract}

\section{INTRODUÇÃo Estudos de distribuição granulométrica} dos sedimentos que revestem os fundos dos corpos d'água costeiros são numerosos, tanto na literatura internacional como na brasileira. Entretanto parecem ser raros os tra. balhos que indiquem a permanência, ou seja, a validade no tempo, das fácies determinadas.

Este trabalho visa caracterizar a faciologia do fundo da Baía de Santos, conforme foi determinada por duas amostragens defasadas de sete anos, bem como a da região adjacente à Ponta de Itaipu (limite oeste da baía), neste caso mediante cinco amostragens defasadas ao longo de um ano.

No primeiro caso, procurou-se a variação das fácies sedimentares por um período mais longo de tempo enquanto, no segundo caso, nossa atenção esteve voltada para as variações de mais curta duração, particularmente as decorrentes das mudanças sazonais no regime hidrodinâmico.

MATERIAIS E MÉTODOS As amostragens na baía foram efetuadas em 1973 e em 1980, ambas ao final do inver. no e início da primavera. A mesma rede de amostragem foi sugerida para as duas campanhas e a localização das estações de amostragem é mostrada na figura 1 . Nas adjacências da Ponta de Itaipu foi usada a rede da figura 2 com coletas de amostras em dezembro de 1974, janeiro, março, agosto e outubro de 1975 , que cobre aproximadamente, o período de um ano.

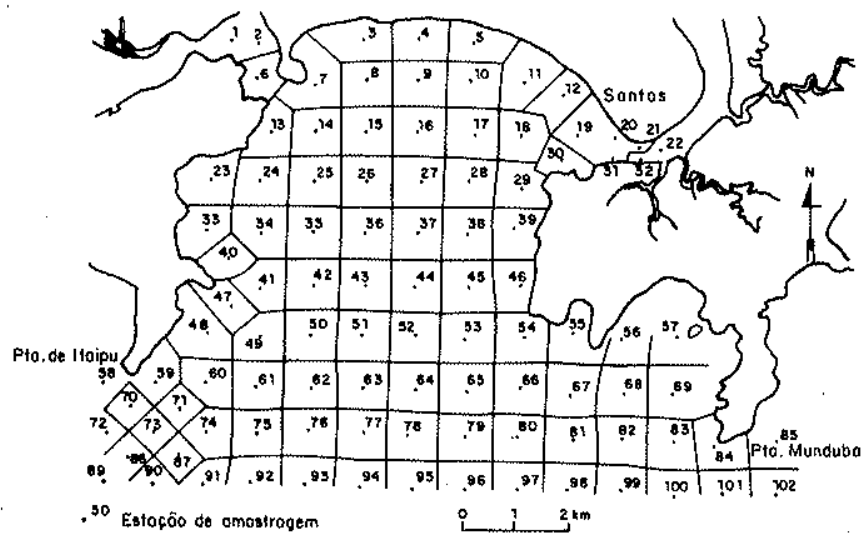

Figura 1 -Estaf̧ões de amostragem de sedimentos de superficie de fundo na Baía de Santos

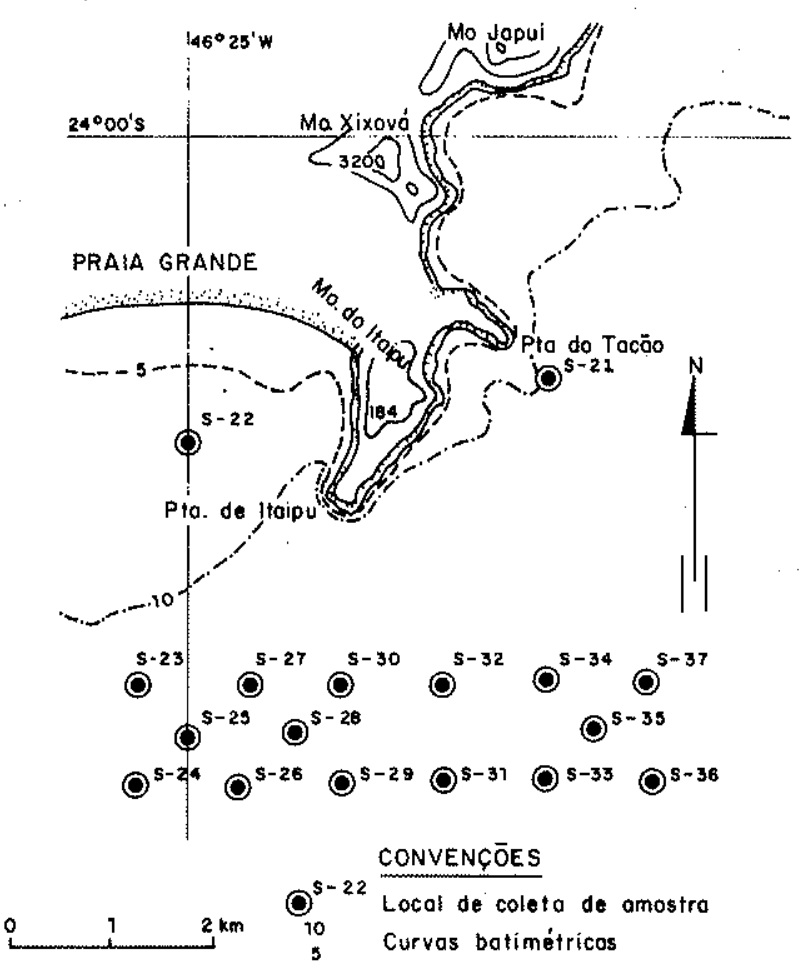

Figura 2 - Localização das estações de coleta de amostras de controle nas adjacências da Ponta de Itaipu

As amostras foram coletadas com amostrador de mandíbula do tipo Van Veen, utilizando-se o barco Emilia do Instituto Oceanográfico da Universidade de São Paulo. A localização das estações de amostragem foi feita por meio de sextante, por aproximação sucessiva, balizada por pontos notáveis assinalados nas cartas náuticas.

O material assim coletado foi submetido à análise granulométrica convencional, con peneiramento a seco para as areias em intervalos de $1 / 2 \phi$ e pipetagem em intervalos de $1 \phi$ para siltes e argilas. Diâmetros médios, adiante referidos, foram calculados segundo a fórmula de Folk \& Ward (1957).

\footnotetext{
* Instituto de Pesquisas Tecnológicas do Estađo de São Paulo-IPT DMGA Cx. Postal 7141, CEP 01000, São Paulo, SP, Brasil; Instituto de Geociências e Ciências Exatas, UNESP, CEP 13.500, Rio Claro, SP, Brasil

* * Instituto de Pesquisas Tecnológicas do Estado de São Paulo-IPT DGMA Cx. Postal 7141, CEP 01000, São Paulo, SP, Brasil
} 
RESUltados Para a Baía de Santos, tendo como critério de referência o diâmetro médio, a situação de 1973 está representada na Figura 3 e a de 1980, na figura 4. Das 98 estações, os diâmetros médios permanecem os mesmos em 55, aumentaram em 22 e diminuíram em 21 (Fig. 5).

Os resultados obtidos para as adjacências da Ponta de Itaipu acham-se resumidos na tabela 1 . No conjunto, mostram pequenas variações, geralmente oscilações para intervalos granulométricos próximos.

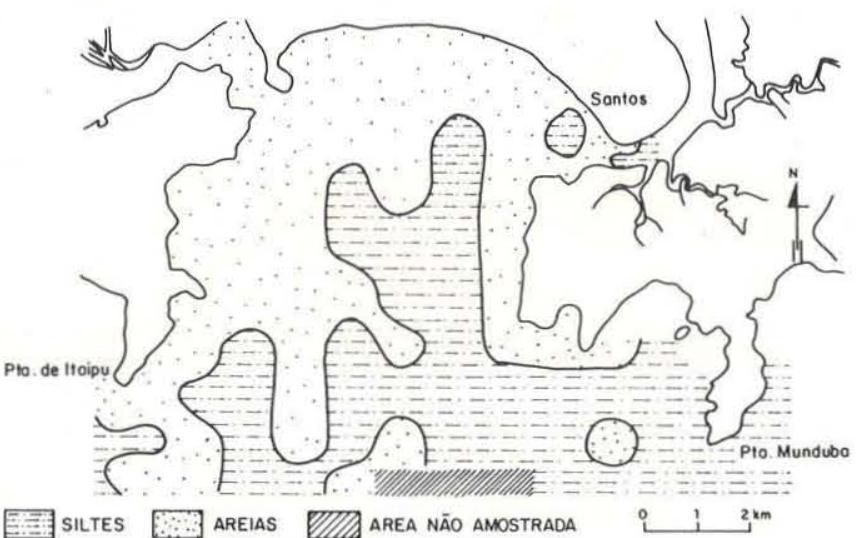

Figura 3 - Distribuição do diâmetro médio em 1973

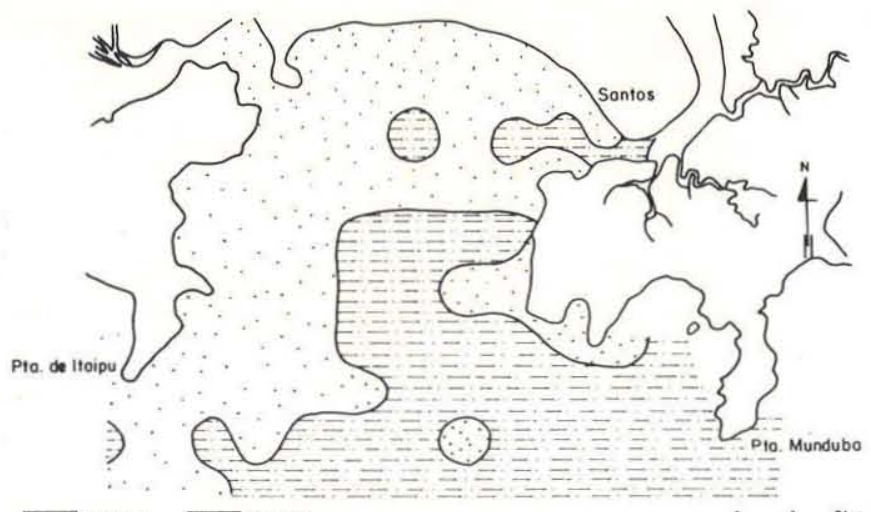

EE:A SILTES $\square$ AREIAS

Figura 4 - Distribuição do diâmetro médio em 1980

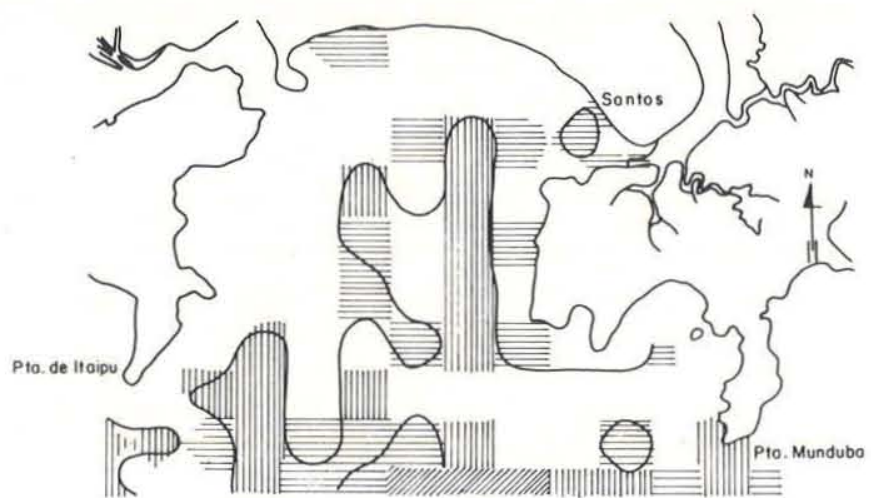

DIĀMETROS MÉDIOS IDENTICOS [IIIIII] AUMENTO DE DIĀMETROS MÉOIOS DIMINUIÇĀO OE DIĀMETROS MÉDIOS UIIA ÁREA NĀO COMPARÁVEL LIMITE SILTE/AREIA ㄴ. $2 \mathrm{~km}$

Figura 5 - Variação da distribuição do diâmetro médio entre 1973 e 1980
Tabela 1 - Variações de diâmetros médios nas adjacências da Ponta de Itaipu

\begin{tabular}{|c|c|c|c|c|c|}
\hline $\begin{array}{l}\text { DATA DE } \\
\text { COLETA }\end{array}$ & AMOSTRA & $\begin{array}{l}\text { Diaimetro } \\
\text { MÉDIO }\end{array}$ & $\begin{array}{l}\text { DATA DE } \\
\text { COLETA }\end{array}$ & AMOSTRA & $\begin{array}{l}\text { DIÄMETRO } \\
\text { MÉDIO }\end{array}$ \\
\hline $11-12 / 11 / 1974$ & S-21 & Arcia m. fina & $11-12 / 11 / 1974$ & S-30 & Areia $m$. fina \\
\hline is.20/01/1975 & S-21A & Areia $m$. fina & $15-20 / 01 / 1975$ & & Areb m. fina \\
\hline $15 / 05 / 1975$ & S-21B & Areia m. fina & $\begin{array}{l}15 / 05 / 1975 \\
\end{array}$ & S. $30 \mathrm{~B}$ & Areb $\mathrm{m}$. fina \\
\hline 13-18/08/1975 & S-21C & Areia m. fina & $13-18 / 08 / 1975$ & S-30C & Sitte fino \\
\hline $20 / 10 / 1975$ & S-21D & Areia $m$. fina & $20 / 10 / 1975$ & S. 300 & Areia m. fina \\
\hline $11-12 / 11 / 1974$ & s-22 & Areia $m$. fina & $11-12 / 11 / 1974$ & S-31 & Silte médio \\
\hline $15-20 / 01 / 1975$ & S. $22 \mathrm{~A}$ & Areia $m$. fina & $15-20 / 01 / 1975$ & & \\
\hline $15 / 05 / 1975$ & S. $22 \mathrm{~B}$ & Silte grosso & $15 / 05 / 1975$ & S-31B & Silte médio \\
\hline $13-18 / 08 / 1975$ & $\mathrm{~S} 22 \mathrm{C}$ & Areia $\mathrm{m}$. fina & $13-18 / 08 / 1975$ & S-3ic & Silte fino \\
\hline $\begin{array}{l}20 / 10 / 1975 \\
\end{array}$ & S.22D & Areia m. fina & $20 / 10 / 1975$ & S.31D & Silte fino \\
\hline $11-12 / 11 / 1974$ & S.23 & Silte $\mathrm{m}$. fino & $11-12 / 11 / 1974$ & S.32 & Silte médio \\
\hline $15-20 / 01 / 1975$ & S.23A & Silte fino & $15.20 / 11 / 1975$ & & Areia $m$. fina \\
\hline $\begin{array}{l}15 / 05 / 1975 \\
\end{array}$ & S-23B & Silte fino & $\begin{array}{l}15 / 05 / 1975 \\
\end{array}$ & S. $32 B$ & Areia m. fina \\
\hline $13-18 / 08 / 1975$ & S. $23 \mathrm{C}$ & Silte fino & $13-18 / 08 / 1975$ & S. $32 \mathrm{C}$ & Silte médio \\
\hline $20 / 10 / 1975$ & S-23D & Silte médio & $20 / 10 / 1975$ & S-32D & Areia $m$. fina \\
\hline $11-12 / 11 / 1974$ & S.24 & Silte médio & $11-12 / 11 / 1974$ & S-23 & Areia $m$. fina \\
\hline is. $20 / 01 / 1975$ & & & $15-20 / 01 / 1975$ & & \\
\hline $15 / 05 / 1975$ & S. $24 \mathrm{~B}$ & Areia $m$. fina & $15 / 05 / 1975$ & S. 33 B & Silte médio \\
\hline $13-18 / 08 / 1975$ & S. $24 \mathrm{C}$ & Areia m. fina & $13-18 / 08 / 1975$ & & Silte médio \\
\hline $20 / 10 / 1975$ & S-24D & Areia $m$. fina & $20 / 10 / 1975$ & S.33D & Silte médio \\
\hline $11 \cdot 12 / 11 / 1974$ & S-25 & Silte fino & $11-12 / 11 / 1974$ & S-34 & Arcia m. fina \\
\hline $15-20 / 01 / 1974$ & S. $25 \mathrm{~A}$ & Areia $\mathrm{m}$. fina & $15-20 / 01 / 1975$ & & Arcia $m$. fina \\
\hline $15 / 05 / 1975$ & S-25B & Areia $\mathrm{m}$. fina & $15 / 05 / 1975$ & S-34B & Areia m. fi \\
\hline $13-18 / 08 / 1975$ & S. $25 \mathrm{C}$ & Areia $\mathrm{m}$. fina & $13-18 / 08 / 1975$ & S- $34 \mathrm{C}$ & Arcia $m$. fina \\
\hline $20 / 10 / 1975$ & S-25D & Areia $\mathrm{m}$. fina & $20 / 10 / 1975$ & S.34D & Arcia $m$. fina \\
\hline $11-12 / 11 / 1974$ & S-26A & Areia m. fina & $11-12 / 11 / 1974$ & S-35 & Areia $m$. fina \\
\hline $15-20 / 01 / 1975$ & S- $26 \mathrm{~A}$ & & is-20/01/1975 & S. $35 \mathrm{~A}$ & Silte fino \\
\hline $15 / 05 / 1975$ & $S-26 B$ & Silte médio & $15 / 05 / 1975$ & S. $35 \mathrm{~B}$ & Silte fino \\
\hline $13-18 / 08 / 1975$ & S-26C & Silte médio & $13-18 / 08 / 1975$ & s.35c & Arcia $m$. fina \\
\hline $20 / 10 / 1975$ & S-26D & Areia $m$. fina & $20 / 10 / 1975$ & S.35D & Areia $m$. fina \\
\hline $11-12 / 11 / 1974$ & S-27 & Silte médio & $11-12 / 11 / 1974$ & S-36 & Silte grosso \\
\hline $15-20 / 11 / 1975$ & S-27A & Areia $m$. fina & $15-20 / 01 / 1975$ & & Areia $\mathrm{m}$. fina \\
\hline $15 / 05 / 1975$ & S- 278 & Silte $m$. fino & $15 / 05 / 1$ & & Silte grosso \\
\hline $13-18 / 08 / 1975$ & S-27C & Areia m. fina & $13-18 / 08 / 1975$ & S. $36 \mathrm{C}$ & Areia $\mathrm{m}$. fina \\
\hline $20 / 10 / 1975$ & S-27D & Area $m$. fina & $20 / 10 / 1975$ & S.36D & Arcia $m$. fina \\
\hline $11-12 / 11 / 1974$ & & & $11-12 / 11 / 1974$ & S.37A & \\
\hline $15-20 / 01 / 1975$ & S.29A & Areia m. fina & $15-20 / 01 / 1975$ & & Silte grosso \\
\hline $15 / 05 / 1975$ & S- $29 B$ & Silte médio & $15 / 05 / 1975$ & S. $37 \mathrm{~B}$ & Silte grosso \\
\hline $13-18 / 08 / 1975$ & S-29C & Silte méd io & $13-18 / 08 / 1975$ & S-37c & Areia m. fina \\
\hline $20 / 10 / 1975$ & S.29D & Silte fino & $20 / 10 / 1975$ & S. 37D & Arcia m. fina \\
\hline
\end{tabular}

DISCUSSÃo A Baía de Santos mostra, nas situações de 1973 e 1980, configurações semelhantes de suas fácies de superfície de fundo, que caracterizam dois setores distintos de circulação: um a oeste (marcado por areias) e outro a leste (denotado por finos), conforme o padrão interpretado por Fúlfaro \& Ponçano (1976).

Algumas variações de limites foram observadas e podem ser analisadas sob dois pontos de vista principais: da variação do aporte continental e da mobilidade das areias da plataforma.

Assim, admite-se que o setor leste da baía receba contribuição de finos provenientes do estuário, que se depositariam em parte na baía e, em parte, seguiriam rumo a Ponta Munduba (Fúlfaro \& Ponçano 1976). Além deles, há que considerar os finos provenientes das desembocaduras de outros rios, a sudoeste de Santos, que perlongam a costa por meio de corrente sudoeste-nordeste (Ponçano 1976). Em decorrência variações sazonais de descargas fluviais podem atuar no sentido de alterar os limites entre finos e grossos no interior da baía.

Por outro lado, é preciso considerar a possibilidade de os próprios fundos arenosos estarem migrando, o que também poderia explicar, ao menos pro-parte, as alterações dos acima mencionados limites.

Nesse sentido, os pesquisadores da Bahia, em sua reconstituição paleoambiental da Baía de Todos os Santos (Bittencourt et al. 1976), chamam a atenção para a possibilidade de serem as areias do fundo daquele corpo d'água reliquiares, hipótese que cabe considerar no caso de Santos.

Esta questão é da maior importância na delimitação do uso que se pode fazer das fácies sedimentares como indicadores do presente nível de energia do meio de transporte e coloca dúvida se os fundos arenosos da Baía de Santos correspondem à dinâmica sedimentar atual, conforme postula a literatura prévia (Fúlfaro \& Ponçano 1976). 
Ensaios de espalhamento de radioisótopos realizados no setor oeste da Baía de Santos (Nuclebrás 1981) mostram efetivo transporte de material arenoso, da ordem de 10 $\mathrm{kg} / \mathrm{m}$.dia no verão ao $200 \mathrm{~kg} / \mathrm{m}$.dia no inverno. O espalhamento se processaria para norte e leste, ou seja, conforme sugeriram Fúlfaro \& Ponçano (1976) a partir da faciologia.

Parece possível então admitir que, embora parte das areias da Baía de Santos possa ser reliquiar, sejam elas efeti. vamente movimentadas por correntes atuais, correspondendo então às regiões de maior energia de transporte.

CONCLUSÃo Neste estudo de variações temporais de fácies de superfície de fundo, os padrões determinados na
Baía de Santos, por campanhas defasadas de sete anos, e nas adjacências da Ponta de Itaipu, em cinco campanhas defasadas ao longo de um ano, mostraram-se relativamente cosntantes. E possivel que, dadas as grandes dimensões da baía, suas fácies de fundo estejam ainda refletindo fases passadas da evolução da plataforma continental. Entretanto a distribuição de finos concorda com os processos atuais de movimentação de sedimentos, quais sejam a corrente costeira sudoeste-nordeste e o fluxo da desembocadura do estuário, na porção leste da baía. No que se refere às areias, por analogia com outros setores da costa, há possibilidade de se tratar de sedimentos reliquiares; sua movimentação por processos atuais acha:se, porém, provada por medidas diretas de espalhamento de radioisótopos.

\section{REFERÊNCIAS BIBLIOGRÁFICAS}

BITTENCOURT, A.C. da S.P.; FERREIRA, Y.A.; NAPOLI, E. 1976 - Alguns aspectos da sedimentação na Baía de Todos os Santos, Bahia. Rev. Bras. Geoc., 6 (4).

FOLK, R.L. \& Ward, W.C. - 1957 - Brazos River bar: a study in significance of grain size parameters. J. Sedim. Petrol, 27:2-26.

FULFARO, V.J. \& PONÇANO, W.L. - 1976 - Sedimentação atual do estuário e baía de Santos: um modelo geológico aplicado a projetos de expansão da zona portuária. In: CONG. BRAS. GEOL. de ENG., 1, Rio de Janeiro, 1976. Anais... Rio de Janeiro, ABGE. v. 2, p. 67-90.
NUCLEBRĀS - 1981 - Movimentaçăo de sedimentos de fundo na bata de Santos. Estudos com o traçador injetado no verão. Belo Horizonte, Centro de Desenvolvimento de Tecnologia Nuclear. Divisão de Engenharia Ambiental (DERL. PD-035/81).

PONÇANO, W.L. - 1976 - Características gerais da seđimentação e correntes costeiras entre Santos e Cananéia. Noticia geomorfologica, Campinas, 16 (31): 107-111.

MANUSCRITO

Recebido em 20 de março de 1986 Revisão aceita em 02 de junho de 1986

MACAMBIRA, J.B. - 1985 - Geologia e Ocorrências Minerais do Braquianticlinal de Lontra, GO. Rev. Bras. Geoc. $15(2)$, p. 174. Na seção Resumos de Teses, o nome do autor apareceu incorretamente; onde está JOSÉ BUENANO MACAMBIRA, leia-se JOEL BUENANO MACAMBIRA. 